\title{
LOCALIZATION OF PLASMON UNDER NONLINEAR INTERACTION OF WHISPERING-GALLERY MODES WITH ELECTRON PLASMA OF SPHERICAL MICROCAVITY
}

\author{
V.V. Kabanov and V.A. Sobolevsky \\ Institute of Physics, National Academy of Sciences of Belarus, F. Skaryna Ave. 68, 220072 Minsk, Republic of Belarus \\ E-mail: v.kabanov@ifanbel.bas-net.by
}

Received 26 October 2004

\begin{abstract}
Self-localization mechanisms and conditions of laser radiation in a cold collisionless plasma of spherical microcavity have been studied taking into account the striction nonlinearity and the relativistic effects connected with the stimulated oscillatory movement of electrons. A spherical plasmon localized close to the centre of microcavity has been shown to excite under nonlinear interaction of whispering-gallery modes with electron plasma. It is determined that the realization of self-localized spherical structures of electromagnetic field and spherical plasmons inside the microcavity filled with electron plasma is caused by manifestation of relativistic nonlinearity of the medium.
\end{abstract}

Keywords: plasmon, nonlinear interaction, microresonator, whispering-gallery modes

PACS: 42.65.Jx, 42.65.Tg

\section{Introduction}

Considerable interest in the problem of nonlinear interaction of laser radiation with spherical particles of micron size is shown due to the fact that nonlinear optical effects can be realized with the use of radiation having a relatively low intensity $[1,2]$. In this case, lasing can be obtained as a result of the total internal reflection of radiation from the inside of a microparticle [3], in the form of a set of densely packed whispering-gallery modes. The Q-factor of such a microcavity is several order of magnitude higher than Q-factor of conventional open laser resonators [4]. Beside the known nonlinear effects in this case $[5,6]$, the self-localization of electromagnetic radiation in a form of a structure resonance inside a microcavity is of great importance. The possibility of realization of the spherical structure resonance was investigated for the first time in a medium with a Kerr nonlinearity [7]. An important condition of formation of such structure is the negativity of the initial permittivity of the medium, which allows a suggestion that such structures can exist in microcavities filled with overdense plasma.

The present paper studies the mechanisms and conditions of self-localization of an electromagnetic radiation in the collisionless electron plasma of the spherical microcavity. The nonlinear interaction of the electro- magnetic radiation with a cold plasma was investigated taking into account the striction nonlinearity and the relativistic effects arising as a result of the induced oscillatory motion of electrons. It is shown that the nonlinear interaction of the whispering-gallery modes with a cold electron plasma results in the appearance of the spherical plasmon localized near the centre of the microsphere. It has been established that the relativistic nonlinearity of the medium is the main condition for the formation of self-localized spherical structures of the electromagnetic field and spherical plasmons inside the microcavity filled with a cold electron plasma.

\section{Theoretical model}

The basic equations describing the interaction of an electromagnetic radiation with a collisionless electron plasma are Maxwell equations and the relativistic motion equation for electrons [8-12]. On the introduction of vector $\mathbf{A}$ and scalar $\varphi$ potentials describing the characteristics of electromagnetic field $\left(\mathbf{E}=c^{-1} \partial_{t} \mathbf{A}-\nabla \varphi\right.$, $\mathbf{B}=\nabla \times \mathbf{A})$, the following system can be obtained from the Maxwell equations:

$$
\begin{gathered}
\left(\operatorname{rot} \operatorname{rot}+c^{-2} \partial_{t}^{2}\right) \mathbf{A}=4 \pi n e c^{-1} \mathbf{v}-c^{-1} \nabla \partial_{t} \varphi, \\
c^{-1} \nabla \partial_{t} \mathbf{A}+\Delta \varphi=-4 \pi e\left(n-n_{0}\right) .
\end{gathered}
$$


Equations (1) are closed by the relativistic equation for the electron motion:

$$
\left(\partial_{t}+\mathbf{v} \nabla\right) \mathbf{p}=-e c^{-1} \partial_{t} \mathbf{A}-e \nabla \varphi+e c^{-1} \mathbf{v} \times[\nabla \times \mathbf{A}],
$$

where $\mathbf{v}, m_{0}$, and $\mathbf{p}=m_{0} \gamma \mathbf{v}$ are the velocity, rest mass, and the momentum of electrons, respectively, and $\gamma=\left(1-\mathbf{v}^{2} / c^{2}\right)^{-1 / 2}$ is a relativistic factor. Using the vector identity $(\mathbf{v} \nabla) \mathbf{p}=m_{0} c^{2} \nabla \gamma-\mathbf{v} \times[\nabla \times \mathbf{p}]$, we write Eq. (2) as

$$
\partial_{t} \mathbf{p}=-e c^{-1} \partial_{t} \mathbf{A}-e \nabla \varphi-m_{0} c^{2} \nabla \gamma .
$$

The first two terms in the right-hand side of Eq. (3) represent the Coulomb force. The last term defines the ponderomotive force $\mathbf{F}_{\text {pond }}=-m_{0} c^{2} \nabla \gamma$ which is an expression of the magnetic force acting upon the electrons. The nonrelativistic ponderomotive force was derived in [10] as a Lorentz force averaged over the highfrequency field oscillations, which acts on the electrons in a weakly inhomogeneous electromagnetic field. The action of the ponderomotive force in spatially inhomogeneous electromagnetic wave results in the redistribution of electron plasma density, and since this force is a potential force $\left(\varphi_{\text {pond }}=-m_{0} c^{2} e^{-1} \gamma\right)$, it is convenient to relate it to the scalar potential, thus defining calibration of the potentials in a following way:

$$
\mathbf{A}=-c e^{-1} \mathbf{p}, \quad \varphi=-m_{0} c^{2} e^{-1} \gamma .
$$

Eqs. $(1,3)$ describe the motion of electrons in the presence of self-consistent field of the incident wave and the field induced by the plasma electrons.

The interaction of electron plasma with a spatially inhomogeneous electromagnetic wave will be considered in a quasi-static approximation where the transient processes related to the redistribution of electron plasma density are quite rapid compared to the characteristic time of change in the spatial structure of the field. In this case the distribution of the electron density at each instant is assumed to be steady-state; ponderomotive forces are balanced by the electrostatic forces caused by the redistribution of plasma density. In this case, the electrostatic potential may be taken to be equal to the time-averaged ponderomotive potential $\varphi=\left\langle\varphi_{\text {pond }}\right\rangle_{t}=-m_{0} c^{2} e^{-1}\langle\gamma\rangle_{t}, \partial_{t} \varphi=0$, and the vector potential is transverse $(\nabla \mathbf{A}=0)$ as for the initial wave. Thus, the above-mentioned potential calibration (4) makes it possible to uniquely relate, in the quasi-static approximation, the electromagnetic potentials to the physical components of the system under consideration: the vector potential decribes the transverse electromagnetic wave propagating in the plasma, and the scalar potential is related to the electrostatic field induced by the redistributions of charge density.

With account for the assumptions made, system (1) can be represented in the form

$$
\begin{gathered}
\left(\Delta-c^{-2} \partial_{t}^{2}\right) \mathbf{A}=4 \pi n e c^{-1} \mathbf{v}, \\
\Delta \varphi=-4 \pi e\left(n-n_{0}\right) .
\end{gathered}
$$

Then, Eqs. (4) and (6) give explicit expressions for the velocity and concentration of the electrons:

$$
\begin{gathered}
n(\mathbf{a})=n_{0}\left(1+c^{2} \omega_{p 0}^{-2} \Delta \gamma\right), \\
\mathbf{v}(\mathbf{a})=-c \gamma^{-1} \mathbf{a},
\end{gathered}
$$

where the relativistic factor has the form $\gamma=\sqrt{1+\mathbf{a}^{2}}$, and the potentials are renormalized as $\mathbf{a}=e m_{0}^{-1} c^{-2} \mathbf{A}$ and $\Phi=e m_{0}^{-1} c^{-2} \varphi$. The right-hand side of Eq. (5) describes the interaction of plasma electrons with the electromagnetic field. The current $\mathbf{j}=n e \mathbf{v}$ present here depends nonlinearly on the vector potential a because of the redistribution of electron plasma density (see Eq. (7)) in spatially inhomogeneous electromagnetic field (striction nonlinearity) and the manifestation of relativistic effects in $n(\mathbf{a})$ and $\mathbf{v}(\mathbf{a})$ (relativistic nonlinearity). From Eqs. (4), (5), and (6) the following nonlinear wave equation for vector potential can be derived:

$$
\left(\Delta-c^{-2} \partial_{t}^{2}-k_{p}^{2} \gamma^{-1}\left(1+k_{p}^{-2} \Delta \gamma\right)\right) \mathbf{a}=0,
$$

where $k_{p}=\omega_{p 0} / c$, and $\omega_{p 0}^{2}=4 \pi n_{0} e^{2} / m_{0}$ is plasma frequency of the undisturbed plasma.

Futher analysis will be made for the standing monochromatic wave with a frequency $\omega: \mathbf{a}=\mathbf{a}_{0}(\mathbf{r}) \cos \omega t$, where $\mathbf{a}_{0}$ is an amplitude of the vector potential. On neglecting the generation of higher harmonics and taking into account the self-action in the nonlinear medium, Eq. (9) can be written as

$$
\left(\Delta+k_{0}^{2}-k_{p}^{2} F(\mathbf{r})\right) \mathbf{a}_{0}=0,
$$

where

$$
\begin{aligned}
& F(\mathbf{r})=\frac{\omega}{\pi} \int_{0}^{2 \pi / \omega} \frac{\cos ^{2} \omega t}{\sqrt{1+\mathbf{a}_{0}^{2} \cos ^{2} \omega t}} \\
& \times\left(1+k_{p}^{-2} \Delta \sqrt{1+\mathbf{a}_{0}^{2} \cos ^{2} \omega t}\right) \mathrm{d} t .
\end{aligned}
$$

In the case of the amplitude $\left|\mathbf{a}_{0}\right| \ll 1$, on expanding the nonlinear terms in Eq. (10) into a series and retaining 
the first order of the relativistic amendment, the following equation can be obtained:

$$
\left(\Delta+k^{2}\right) \mathbf{a}_{0}=\frac{3}{8} \mathbf{a}_{0}\left(\Delta-k_{p}^{2}\right) \mathbf{a}_{0}^{2},
$$

where $k^{2}=k_{0}^{2}-k_{p}^{2}$. The right-hand side of Eq. (11) describes the nonlinear interaction of the electromagnetic radiation with the electron plasma: the first term is caused by the striction nonlinearity and the second term is responsible for the relativistic effects.

Let us consider the behaviour of an electrically transverse (TE) electromagnetic radiation in a spherical microcavity filled with a collisionless electron plasma. In the general case it can be represented as an expansion in terms of the vector spherical modes $\mathbf{M}_{n m}(r, \theta, \varphi)=S_{n m}(r) \boldsymbol{\Theta}_{n m}(\theta, \varphi)$, which form a complete set of orthogonal functions:

$$
\begin{gathered}
\mathbf{a}_{0}=\sum_{n m} S_{n m}(r) \boldsymbol{\Theta}_{n m}(\theta, \varphi), \\
S_{n m}(r)=\int_{\Omega} \mathbf{a}_{0} \boldsymbol{\Theta}_{n m}^{*}(\theta, \varphi) \mathrm{d} \Omega, \\
n \in[0, \infty), \quad m \in[-n, n],
\end{gathered}
$$

where

$$
\begin{aligned}
\boldsymbol{\Theta}_{n m}(\theta, \varphi)= & N_{n m} \mathrm{e}^{\mathrm{i} m \varphi}\left(\mathrm{i} m P_{n|m|}(\cos \theta) \sin ^{-1} \theta \mathbf{e}_{\theta}\right. \\
& \left.-\partial_{\theta} P_{n|m|}(\cos \theta) \mathbf{e}_{\varphi}\right)
\end{aligned}
$$

is the angular part, and the radial functions $S_{n m}(r)$ are the desired quantities. For example, the radial functions in the case of linear microcavity are spherical Bessel functions $j_{n}(k r)$ [13]. For the nonlinear problem, $S_{n m}(r)$ have to be determined from a corresponding system of equations, and in the general case they are complex quantities, $S_{n m}=S_{n-m}^{*}$.

We expand the right-hand side of nonlinear equation (11) into a series of spherical vector harmonics:

$$
\begin{gathered}
\frac{3}{8} \mathbf{a}_{0}\left(\Delta-k_{p}^{2}\right) \mathbf{a}_{0}^{2}= \\
\frac{3}{8} \sum_{\frac{i j i^{\prime} j^{\prime}}{k l n m}}\left[g_{n m}^{i j i^{\prime} j^{\prime} k l} S_{n m}-k_{p}^{2} f_{n m}^{i j i^{\prime} j^{\prime} k l} S_{i j} S_{i^{\prime} j^{\prime}} S_{k l}\right] \mathbf{\Theta}_{n m},
\end{gathered}
$$

where $f_{n m}^{i j i^{\prime} j^{\prime} k l}$ is a constant and $g_{n m}^{i j i^{\prime} j^{\prime} k l}$ is a functional of the desired functions $S_{n m}(r)$ :

$$
g_{n m}^{i j i^{\prime} j^{\prime} k l}=
$$

$$
\begin{gathered}
S_{k l} S_{n m}^{-1} \int_{\Omega}\left(\boldsymbol{\Theta}_{k l} \boldsymbol{\Theta}_{n m}^{*}\right) \Delta\left(S_{i j} S_{i^{\prime} j^{\prime}} \boldsymbol{\Theta}_{i j} \boldsymbol{\Theta}_{i^{\prime} j^{\prime}}\right) \mathrm{d} \Omega, \\
f_{n m}^{i j i^{\prime} j^{\prime} k l}=\int_{\Omega}\left(\boldsymbol{\Theta}_{i j} \boldsymbol{\Theta}_{i^{\prime} j^{\prime}}\right)\left(\boldsymbol{\Theta}_{k l} \boldsymbol{\Theta}_{n m}^{*}\right) \mathrm{d} \Omega
\end{gathered}
$$

By making use of the expansion (13) and separating the modes in accordance with their order, the following system of related equations for individual modes can be obtained from Eq. (11):

$$
\begin{gathered}
\Delta \mathbf{M}_{n m}+k^{2} \mathbf{M}_{n m}= \\
\frac{3}{8} \mathbf{M}_{n m} \sum_{i j i^{\prime} j^{\prime} k l}\left(g_{n m}^{i j i^{\prime} j^{\prime} k l}-k_{p}^{2} f_{n m}^{i j i^{\prime} j^{\prime} k l} S_{i j} S_{i^{\prime} j^{\prime}} S_{k l} S_{n m}^{-1}\right) .
\end{gathered}
$$

Separation of variables in Eq. (15) results in a system of equations for radial functions $S_{n m}(r)$ of the interacting modes:

$$
\begin{gathered}
r^{-1} \partial_{r}^{2}\left(r S_{n m}\right)+S_{n m}\left(k^{2}-n(n+1) r^{-2}\right. \\
-\frac{3}{8} \sum_{i j i^{\prime} j^{\prime} k l}\left(g_{n m}^{i j i^{\prime} j^{\prime} k l}-k_{p}^{2} f_{\left.\left.n m^{i j i^{\prime} j^{\prime} k l} S_{i j} S_{i^{\prime} j^{\prime}} S_{k l} S_{n m}^{-1}\right)\right)}=0 .\right.
\end{gathered}
$$

In the case of a single mode in a medium, the system (16) reduces to the form

$$
\begin{gathered}
r^{-1} \partial_{r}^{2}\left(r S_{n m}\right)+S_{n m}\left(k^{2}-n(n+1) r^{-2}\right. \\
-\frac{3}{8} \int_{\Omega}\left(\operatorname{Re}\left(\mathrm{S}_{\mathrm{nm}} \boldsymbol{\Theta}_{\mathrm{nm}}\right) \boldsymbol{\Theta}_{n m}^{*}\right) \\
\times\left(\Delta-k_{p}^{2}\right) \operatorname{Re}\left(S_{n m} \boldsymbol{\Theta}_{n m}\right)^{2} \mathrm{~d} \Omega=0 .
\end{gathered}
$$

It should be noted that Eqs. $(11,15-17)$ describe the interaction of electromagnetic radiation with a cold electron plasma, taking into account both the striction and the relativistic nonlinearities. In spite of the assumption made above in the first approximation for the relativistic effects $\left(\left|\mathbf{a}_{0}\right| \ll 1\right)$, these equations are valid in a quite wide range of radiation intensities, which is important from the practical standpoint. For example, at $\omega=3 \cdot 10^{15} \mathrm{~s}^{-1}$ they describe in a close approximation the nonlinear interaction of an electron plasma with electromagnetic radiation of the intensity up to $I=c^{3} m_{0}^{2} \omega^{2} / 4 \pi e^{2} \approx 8.4 \cdot 10^{18} \mathrm{~W} / \mathrm{cm}^{2}$. At the same time, as it will be shown below, taking into account the relativistic nonlinearity results in qualitatively new behaviour of the system.

\section{Numerical simulation}

The nonlinear interaction of an electromagnetic radiation with a cold electron plasma will be analyzed using a vector spherical mode of a lower order $(n=1$ 


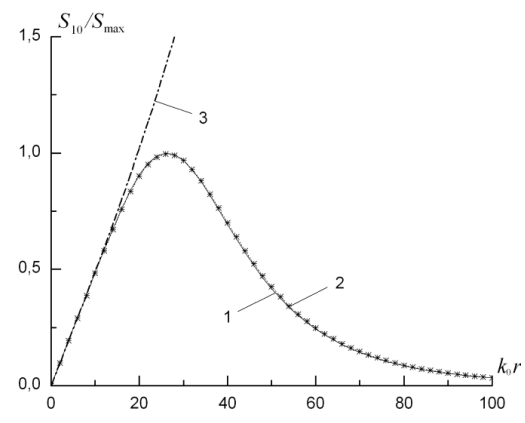

(a)

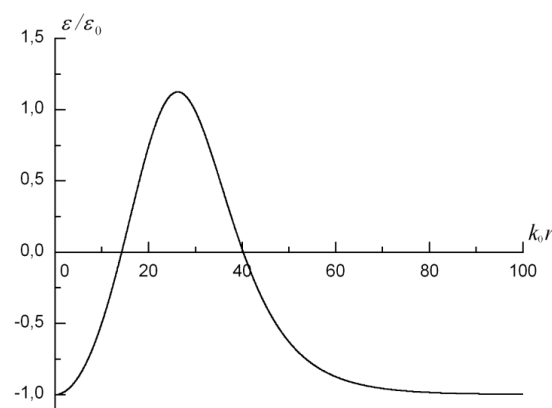

(b)

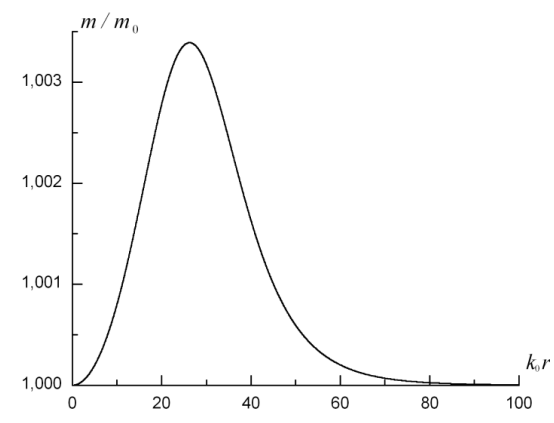

(c)

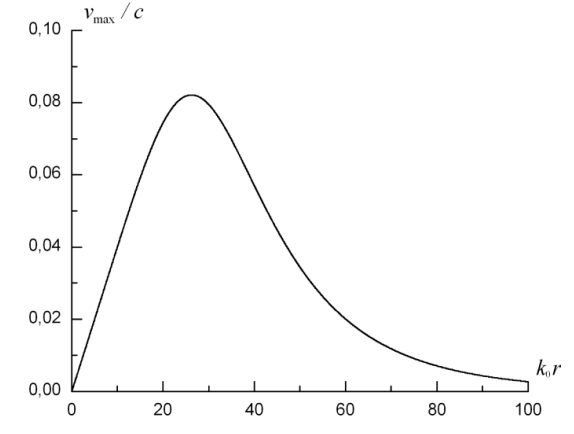

(d)

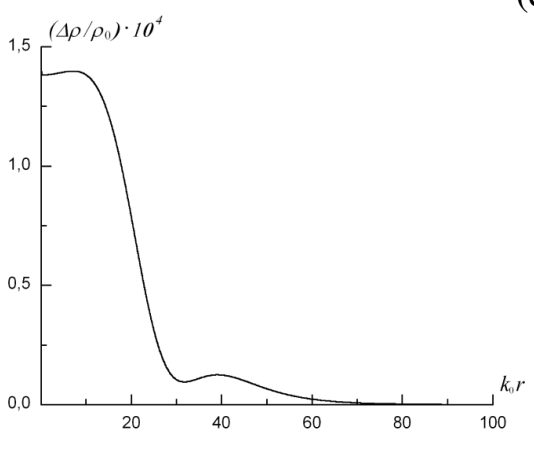

(e)

Fig. 1. Radial distributions of the (a) field, (b) permittivity, (c) mass, (d) electron velocity, and (e) plasma density for $S_{10}$ mode in a microcavity filled with an initially overdense plasma.

and $m=0$ ). In this case, the Eq. (17) yields the following equation for the radial part $S_{10}(r)$ :

$$
\begin{aligned}
& r^{-1} \partial_{r}^{2}\left(r S_{10}\right)+S_{10}\left[k^{2}-2 r^{-2}+0.1125 \pi^{-1}\right. \\
& \left.\quad \times\left(k_{p}^{2}-r^{-1} \partial_{r}^{2}\left(r S_{10}^{2}\right)+S_{10}^{2} r^{-2}\right)\right]=0 .
\end{aligned}
$$

The results of numerical simulation of the process described by Eq. (18) for an overdense plasma $\left(\omega<\omega_{p 0}\right)$ point to the possibility of realization of soliton-like solutions self-localized in a finite volume of a microcavity. The radial distribution of the electric component of the field (Fig. 1(a)) shows its localization close to the centre of the microcavity at a distance of several wavelengths. In this case, the relativistic nonlinearity results in the formation of a layer transparent for electromagnetic radiation near the centre of microcavity. The plasma frequency $\omega_{p}=4 \pi e^{2} \mathrm{n} \mathrm{m}^{-1}$ inside this layer is shifted to the long-wave region of a spectrum so that $\omega_{p}<\omega<\omega_{p 0}$. In this case, the relativistic effect causing a change in the mass $\left(m=\gamma m_{0}\right)$ and velocity of the electrons (Eq. (8)) plays a dominant role. The radial distribution of the permittivity $\varepsilon=1-\left(\omega_{p} / \omega\right)^{2}$ induced by the field (see Fig. 1(b)) shows that in the region of field maximum the permittivity becomes positive. The changes in the mass and the velocity distribution of electrons in the radial direction with taking into account the relativity are shown in Figs. 1(c) and 1(d) respectively.
Thus, the induced spherical layer of plasma transparent for electromagnetic radiation makes possible the existence of the undamped field oscillations inside this layer and the total internal reflection at its border $[14,15]$. The results of numerical simulation of the processes performed with account for only the relativistic nonlinearity (curve 2 in Fig. 1(a)) clearly point to the fact that it is the relativistic nonlinearity that plays a dominant role in the realization of self-localized soliton-like states of the electromagnetic radiation nonlinearly interacting with the cold electron plasma. Taking into account of only the striction nonlinearity in Eq. (18) gives indefinitely increasing solutions (curve 3 in Fig. 1(a)). At the same time, the inclusion of the striction nonlinearity in Eq. (18) determines the existence of a spherical plasmon as a redistribution of the initial equilibrium density of the electron plasma $\Delta \rho$ (Fig. 1(e)), and its localization close to the centre due to the relativistic nonlinearity.

For the case of electron plasma initially transparent for electromagnetic radiation $\left(\omega>\omega_{p 0}\right)$, soliton-like states localized in a finite volume cannot be realized. The radial field distribution shown in Fig. 2 (curve 1) illustrates this situation for the $n=1, m=0$ mode. Curve 2 in Fig. 2 was calculated with taking into account only the striction nonlinearity. In the case of transparent plasma with taking into account of the striction and the relativistic nonlinearities as well as the case 


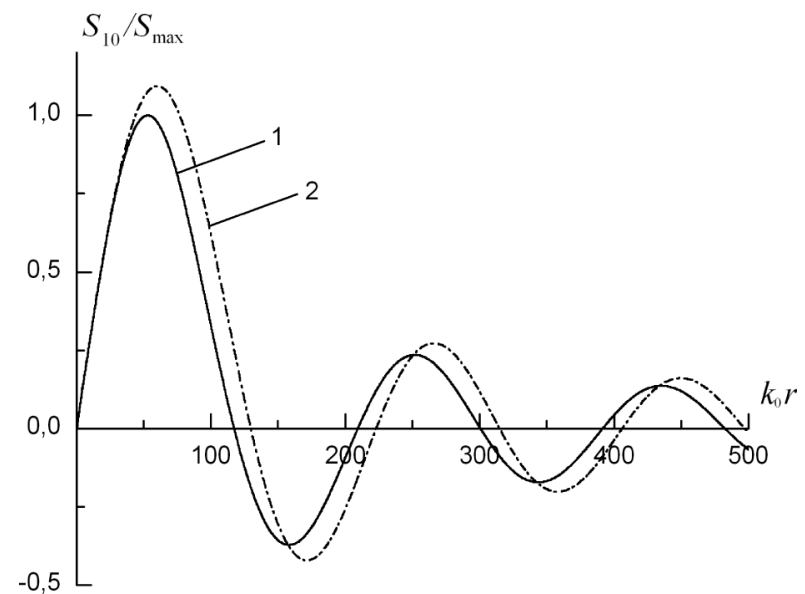

Fig. 2. Radial distribution of the field for $S_{10}$ mode in a microcavity filled with plasma initially transparent for electromagnetic radiation.

of overdense plasma where only the striction nonlinearity was taken into account (curve 3 in Fig. 1(a)) it should be noted that the behaviour of the system does not differ qualitatively from the corresponding solutions of the linear wave equation for an electron plasma.

\section{Conclusion}

A model of an electromagnetic radiation nonlinearly interacting with a cold electron plasma inside a spherical microcavity has been developed with taking into account both the striction and the relativistic nonlinearities. The process of nonlinear interaction was numerically simulated on the basis of the equations set with account of the relativistic effects in the first approximation. This approach can be used in a quite wide range of radiation intensities of up to $\sim 10^{18} \mathrm{~W} / \mathrm{cm}^{2}$, which is important from the practical standpoint. It is shown that soliton-like solutions self-localized in the finite volume of a nonlinear microcavity filled with an initially overdense electron plasma can be realized. It has been established that it is the relativistic nonlinearity that plays a key role in the realization of self-localized states of an electromagnetic radiation. The striction nonlinearity results in the appearance of a spherical plasmon as a redistribution of the equilibrium electron plasma density. At the same time, its localization close to the centre is caused by the relativistic nonlinearity.

\section{References}

[1] S.C. Hill and R.E. Benner, in: Optical Effects Associated with Small Particles, eds. P.W. Barber and
R.K. Chang (World Scientific, Singapore, 1988) pp. 361.

[2] C.G.B. Garrett, W. Kaiser, and W.L. Bond, Stimulated emission into optical whispering modes of spheres, Phys. Rev. 124(6), 1807-1809 (1961).

[3] G. Chen, D.Q. Chowdhury, R.K. Chang, and W.-F. Hsieh, Laser-induced radiation leakage from microdroplets, J. Opt. Soc. Amer. B 10, 620-632 (1993).

[4] M.L. Gorodetsky and V.S. Ilchenko, High-Q optical whispering-gallery microresonators: Precession approach for spherical mode analysis and emission patterns with prism couplers, Opt. Commun. 113, 133143 (1994).

[5] H.-M. Tzeng, K.F. Wall, M.B. Long, and R.K. Chang, Laser emission from droplets at wavelengths corresponding to morphology-dependent resonances, Opt. Lett. 9, 499-501 (1984).

[6] J.-Z. Zhang and R.K. Chang, Generation and suppression of stimulated Brillouin scattering in single liquid droplets, J. Opt. Soc. Am. B 6(6), 151-154 (1989).

[7] V.V. Kabanov, Spherically symmetric structural resonances self-localized in a nonlinear medium, Quantum Electron. 26, 820-821 (1996).

[8] A.B. Borisov, A.V. Borovsky, V.V. Korobkin, A.M. Prohorov, Ch.K. Rouds, and O.B. Shiryaev, Relativistic-strictional self-channeling of intense ultrashort laser pulses in a matter, JETP (in Russian) 101(4), 1132-1153 (1992).

[9] A.N. Akhiezer and R.V. Polovin, On the theory of wave motion of an electron plasma, JETP (in Russian) 30(5), 915-928 (1956).

[10] A.V. Gaponov and N.A. Miller, On the potentional wells for the charged particles in a high-frequency electromagnetic field, JETP (in Russian) 34, 242-243 (1958).

[11] J.H. Marburger and R.F. Tooper, Nonlinear optical standing waves in overdense plasmas, Phys. Rev. Lett. 35(15), 1001-1004 (1975).

[12] C.J. McKinstrie and D.F. DuBois, A covariant formalism for wave propagation applied to stimulated Raman scattering, Phys. Fluids 31(2), 278-287 (1988).

[13] J.A. Stratton, Electromagnetic Theory (McGraw-Hill, New York, 1941).

[14] V.V. Kabanov, Three-dimensional soliton selflocalized as a spherically symmetric structural resonance of electromagnetic radiation in a nonlinear medium, Lithuanian J. Phys. 39, 275-281 (1999).

[15] V.V. Kabanov, Dynamics of spherically symmetric vortex of laser radiation in a nonlinear medium, Chaos, Solitons Fractals 17, 425-431 (2003). 


\title{
PLAZMONO LOKALIZACIJA ESANT NETIESINEI ŠNABŽDESIO GALERIJŲ MODŲ SĄVEIKAI SU SFERINĖS MIKROERTMĖS ELEKTRONŲ PLAZMA
}

\author{
V.V. Kabanov, V.A. Sobolevsky \\ Fizikos institutas, Minskas, Baltarusija
}

\begin{abstract}
Santrauka
Išstudijuoti lazerio spinduliuotès savilokalizacijos mechanizmai ir sąlygos šaltoje sferinès mikroertmès be susidūrimų plazmoje, atsižvelgiant i strikcijos netiesiškumą ir reliatyvistinius efektus, susijusius su priverstiniu elektronų virpesiniu judèjimu. Parodyta, kad sferinis plazmonas, lokalizuotas arti mikroertmès centro, sužadina-
\end{abstract}

mas, kai šnabždesio galerijụ modos netiesiškai sąveikauja su elektronu plazma. Nustatyta, kad elektromagnetinio lauko savilokalizuotų sferinių struktūrų ir sferinių plazmonų susidarymą mikroertmèje, užpildytoje elektronų plazma, lemia medžiagos reliativystinis netiesiškumas. 